\author{
$F$-monoids* \\ E. Giraldes, Dpto. de Matemática \\ UTAD, 5000 Vila Real/Portugal \\ e-Mail: egs@utad.pt \\ P. Marques-Smith, Centro de Matemática \\ Universidade do Minho, 4700 Braga/Portugal \\ e-mail: psmith@math.uminho.pt \\ H. Mitsch, Fakultät f. Mathematik \\ Universität Wien, 1090 Wien/Austria \\ e-mail: heinz.mitsch@univie.ac.at
}

\begin{abstract}
A semigroup $S$ is called $F$-monoid if $S$ has an identity and if there exists a group congruence $\rho$ on $S$ such that each $\rho$-class of $S$ contains a greatest element with respect to the natural partial order of $S \leq_{S}$ (see [7]). Generalizing results given in [4] and specializing some of [3] five characterizations of such monoids $S$ are provided. Three unary operations *, $\circ$ and - on $S$ defined by means of the greatest elements in the different $\rho$-classes of $S$ are studied. Using their properties a charaterization of $F$ monoids $S$ by their regular part $S^{\circ}=\left\{a^{\circ} \mid a \in S\right\}$ and the associates of elements in $S^{\circ}$ is given. Under the hypothesis that $S^{*}=\left\{a^{*} \mid a \in S\right\}$ is a subsemigroup it is shown that $S$ is regular, whence of a known structure (see [4]).
\end{abstract}

\title{
1 Introduction and summary
}

A semigroup $S$ is called $F$-monoid if $S$ has an identity and if there exists a group congruence $\rho$ on $S$ such that each $\rho$-class of $S$ admits a greatest element with respect to the natural partial order $\leq_{S}$ on $S$ (see [7]):

$$
a \leq_{S} b \text { if and only if } a=x b=b y, x a=a \text { for some } x, y \in S .
$$

This concept generalizes that of an $F$-regular semigroup (see [4]; note that the latter are necessarily monoids) and is a particular case of an $F$-semigroup (see

${ }^{*}$ Research supported by the Portuguese Foundation for Science and Technology through the research program POCTI. 
[3]). All these notions are special instances of generalized $F$-semigroups (see [2]). These are semigroups $S$, on which there exists a group congruence $\rho$ such that the identity $\rho$-class (only) admits a greatest element with respect to $\leq_{S}$, the pivot of $S$. It was noted in [2], that the congruence $\rho$ is equal to the least group congruence on $S$, whence is uniquely determined. Also, every generalized $F$-semigroup $S$ is $E$-inversive, that is, for any $a \in S$ there exists some $x \in S$ such that $a x \in E_{S}$ (see [9], [10]). If $S$ has an identity then $S$ is $E$-unitary, i.e., if $e, e a \in E_{S}$ or $e, a e \in E_{S}$ then $a \in E_{S}$ (see[2]). Therefore, we are dealing with particular $E$-inversive, $E$-unitary monoids. The existence of an identity element in a semigroup $S$ has a strong impact on the structure of $S$. This observation is again corroborated in the theory of $F$-monoids (compared with $F$-semigroups).

In Section 2, several examples of non-regular $F$-monoids are given. In particular, it is shown that adjoining an identity to an $F$-semigroup does not yield an $F$-monoid, in general. A necessary and sufficient condition for this to hold is given. In Section 3, the following characterizations of $F$-monoids are presented: (i) by residuation of the identity, (ii) by the subsets $T(a)=\left\{x \in S: a x a \leq_{S} a\right\}$, $a \in S$, (iii) by the maximal elements of $\left(S, \leq_{S}\right)$ and (iv) by means of an additional unary operation satisfying certain axioms. Here a new description of $E$-inversive semigroups proves useful. Three unary operations $*$ and $\circ$ (already defined in [4]) and - are considered in Section 4. Several properties of them are proved which are used in the following. In particular, it is shown that for an $F$ monoid $S$, the set $S^{\circ}=\left\{a^{\circ} \in S \mid a \in S\right\}$ forms an $F$-regular subsemigroup of $S$ (the structure of which was studied in [4]). In Section 5 , by means of the regular part $S^{\circ}$ of an $F$-monoid $S$ a characterization of $F$-monoids is given observing that $S$ consists of the associates of elements in $S^{\circ}$. Concerning the second unary operation ${ }^{*}$, the set $S^{*}=\left\{a^{*} \in S \mid a \in S\right\}$ does not form a subsemigroup, in general (see [4]). If this is the case then $S$ is called an $F$-*-monoid; this class of monoids is considered in Section 6 . It turns out that an $F$-*-monoid is necessarily regular, whence by [4] of a known structure.

\section{Examples}

(1) Every regular $F$-semigroup is an $F$-monoid (by [2], Theorem 3.14). The class of $F$-regular semigroups was studied in [4] where also a representation theorem was proved. Hence in the following, only non-regular $F$-monoids will be considered. On the other hand, there are $F$-semigroups without identity: let $S=\{0,1, a\}$ be the inflation (see [11]) of the semilattice $Y: 0<_{Y} 1$, where $a^{2}=a \cdot 1=1 \cdot a=1$ and $a \cdot 0=0 \cdot a=0$; then $S$ is an $F$-semigroup without identity and with pivot $\xi=a$ (by [2], Theorem 3.5). See also the semigroups given in Remark (3) following Corollary 6.2 in [3].

(2) Let $S=\left[Y ; S_{\alpha}, \varphi_{\alpha, \beta}\right]$ be a strong semilattice of trivially ordered monoids such that: $(a)\left(Y, \leq_{Y}\right)$ is a finite chain, $(b)$ each $\varphi_{\alpha, \beta}$ is injective, $(c)$ for every $a \in S, a \in S_{\alpha}$ say, there exist $\beta \leq_{Y} \alpha$ and $x \in S_{\beta}$ with $\left(a \varphi_{\alpha, \beta}\right) x \in E_{S_{\beta}}$. Then conditions (i) and (ii) in Corollary 6.6 of [3] are evidently satisfied; also (iii) holds: let $a, b \in S$ be maximal in $\left(S, \leq_{S}\right), a \in S_{\alpha}, b \in S_{\beta}$ say. If $\alpha=\beta$ then 
$a \varphi_{\alpha, \gamma} \neq b \varphi_{\beta, \gamma}$ for every $\gamma \leq_{Y} \alpha=\beta$ by (b). If $\alpha \neq \beta$ then $\alpha<_{Y} \beta$, say. Assume that $a \varphi_{\alpha, \gamma}=b \varphi_{\beta, \gamma}$ for some $\gamma \leq_{Y} \alpha<_{Y} \beta$.Then $a \varphi_{\alpha, \gamma}=b\left(\varphi_{\beta, \alpha} \circ \varphi_{\alpha, \gamma}\right)=$ $\left(b \varphi_{\beta, \alpha}\right) \varphi_{\alpha, \gamma}$, whence by (b), $a=b \varphi_{\beta, \alpha}$. Therefore $a<_{S} b$ (see [8], proof of Theorem 3.8) and $a \in S$ is not maximal in $\left(S, \leq_{S}\right)$ : contradiction.

It follows that $S$ is an $F$-monoid with $1_{\omega} \in S_{\omega}$ as the identity where $\omega$ denotes the greatest element of $\left(Y, \leq_{Y}\right)$ - note that by the trivial order of $S_{\alpha}$ $(\alpha \in Y), E_{S_{\alpha}}=\left\{1_{\alpha}\right\}$ whence $1_{\omega} \varphi_{\omega, \alpha}=1_{\alpha}$. If at least one $S_{\alpha}(\alpha \in Y)$ is not a group then $S$ is not regular: assume that $a \in S_{\alpha}$ does not have a group-inverse in $S_{\alpha}$, but $a=a x a$ for some $x \in S, x \in S_{\beta}$ say; then $a=a x a \in S_{\alpha \beta}$, whence $\alpha=\alpha \beta$, i.e., $\alpha \leq_{Y} \beta$. Therefore $a=a\left(x \varphi_{\beta, \alpha}\right) a=a y a$ for $y=x \varphi_{\beta, \alpha} \in S_{\alpha}$. Since $E_{S_{\alpha}}=\left\{1_{\alpha}\right\}$ it follows that $a y=y a=1_{\alpha}$ : contradiction. Note that by [8], Corollary $3.9, \leq_{S}$ is compatible with multiplication.

Remarks. (i) Condition (c) is satisfied for example if $S_{\mu}$ is a group where $\mu$ denotes the least element of $\left(Y, \leq_{Y}\right)$.

(ii) If condition (c) is replaced by: "each $S_{\alpha}$ with $\alpha \neq \omega$ in $\left(Y, \leq_{Y}\right)$ is $E$ inversive" then each $S_{\alpha}(\alpha \neq \omega)$ is a group. In fact, $E_{S_{\alpha}}=\left\{1_{\alpha}\right\}$ then implies that for any $a \in S_{\alpha}$ there exists $x \in S_{\alpha}$ such that $a x=1_{\alpha}$. Thus choosing for $S_{\omega}$ a trivially ordered monoid, which is not a group, we shall obtain a non-regular $F$-monoid.

(iii) Examples of trivially ordered monoids are $(\mathbb{N}, \cdot)$ or $\left(\mathbb{N}_{0},+\right)$, more generally, all cancellative monoids. Constructions of trivially ordered monoids were given in [6].

(iv) In (2), $S$ can be replaced by a monoid, which is a strong semilattice of trivially ordered semigroups satisfying (a), (b) and (c) - see Remark 3 to Corollary 3.9 in [8].

As a particular case of (2) we mention

(3) Let $S=\left[Y ; S_{\mu}, S_{\omega} ; \varphi_{\omega, \mu}\right]$ where $Y: \mu<_{Y} \omega, S_{\mu}=G$ is a group, $S_{\omega}=T$ is a subsemigroup of $G$, which is not a subgroup and which contains the identity $1_{G} \in G$, and with $\varphi_{\omega, \mu}: S_{\omega} \rightarrow S_{\mu}, a \varphi_{\omega, \mu}=\bar{a}$, the inclusion mapping. Since both $S_{\mu}$ and $S_{\omega}$ are trivially ordered it follows by (2), that $S$ is a non-regular $F$-monoid with identity $1_{G} \in T$. Note that $S_{\mu}=G$ has to be infinite. If there exists an element $a \in G$ of infinite order one may take $S_{\omega}=T=\left\{1_{G}, a, a^{2}, \ldots\right\}$. For example: $S_{\mu}=(\mathbb{Z},+)$, the group of integers, and $S_{\omega}=\left(\mathbb{N}_{0},+\right)$, the semigroup of natural numbers including 0 .

Generalizing $S_{\mu}=G$ to a Clifford semigroup we obtain

(4) Let $S_{0}=\left[Y ; G_{\alpha}, \varphi_{\alpha, \beta}\right]$ be a Clifford semigroup which is an $F$-semigroup (see [3], Corollary 6.7), and with $Y$ finite. Let $\omega \in Y$ be the greatest element of $\left(Y, \leq_{Y}\right)$ and let $S_{1}=T$ be a subsemigroup of $G_{\omega}$ which is not a subgroup and which contains $1_{\omega} \in G_{\omega}$. Then $S=\left[Z ; G_{\alpha}, S_{1} ; \varphi_{\alpha, \beta}, \varphi_{1, \alpha}\right]$ with $Z=Y^{1}$, $\varphi_{1, \omega}: S_{1} \rightarrow G_{\omega}, a \varphi_{1, \omega}=\bar{a}$, the inclusion mapping, and $\varphi_{1, \alpha}=\varphi_{1, \omega} \circ \varphi_{\omega, \alpha}$ for any $\alpha \in Y$, is a strong semilattice of trivially ordered monoids $S_{1}$ and $G_{\alpha}$ $(\alpha \in Y)$. Again conditions (i) and (ii) in Corollary 6.6 of [3] are satisfied; also (iii) holds:

Let $a, b \in S$ be maximal in $\left(S, \leq_{S}\right)$. If $a, b \in S_{0}$ then $a \in G_{\alpha}, b \in G_{\beta}$ say, and $a \varphi_{\alpha, \gamma} \neq b \varphi_{\beta, \gamma}$ for any $\gamma \leq_{Y} \alpha=\beta$, by [3], Corollary 6.7. If $a, b \in S_{1}$ then $a \varphi_{1, \omega} \neq b \varphi_{1, \omega}$ (since $\varphi_{1, \omega}$ is injective). Since by [2], Corollary 4.7, $\varphi_{\omega, \gamma}$ is 
injective for any $\gamma \in Y$, it follows that $a \varphi_{1, \gamma}=\left(a \varphi_{1, \omega}\right) \varphi_{\omega, \gamma} \neq\left(b \varphi_{1, \omega}\right) \varphi_{\omega, \gamma}=$ $b \varphi_{1, \gamma}$. Finally, if $a \in S_{1}$ and $b \in S_{0}$ then $b \in G_{\alpha}$ say $(\alpha \in Y)$. Assume that $a \varphi_{1, \gamma}=b \varphi_{\alpha, \gamma}$ for some $\gamma \leq_{Y} \alpha<_{Y} 1$. Then $\left(a \varphi_{1, \alpha}\right) \varphi_{\alpha, \gamma}=b \varphi_{\alpha, \gamma}$, hence $a \varphi_{1, \alpha}=b$ (since $\varphi_{\alpha, \gamma}$ is injective, see [3], Proposition 6.4). Therefore $b<_{S} a$ (see [8], proof of Theorem 3.8), which contradicts the maximality of $b \in S_{0}$.

It follows that $S$ is a non-regular $F$-monoid whose identity is $1_{\omega} \in S_{1}$ (see Example (2)).

If a semigroup $S$ has no identity then adjoining one we obtain a monoid $S^{1}$. If $S$ is an $F$-semigroup this procedure does not yield an $F$-monoid, in general, as the following result shows.

Proposition 2.1 Let $S$ be an F-semigroup. Then $S^{1}$ is an F-monoid if and only if the pivot of $S$ is idempotent.

Proof. Necessity. Let $\rho$ (resp. $\sigma$ ) be the defining group congruence on $S$ (resp. on $S^{1}$ ). By the uniqueness of $\rho$ ([2], Theorem 3.6) the restriction of $\sigma$ to $S$ (being a group congruence) is equal to $\rho$. Since 1 is idempotent, $1 \in S^{1}$ belongs to the identity $\sigma$-class $I_{\sigma}$ of the group $S^{1} / \sigma$; hence $I_{\sigma}=I_{\rho} \cup\{1\}$. By [2], Corollary 3.9, $I_{\rho}=E_{S}$ or $I_{\rho}=E_{S} \cup\{a\}$ with $a \notin E_{S}$. Assume that the pivot $\xi$ of $S$ is not idempotent. Then the elements $\xi=a \in I_{\rho} \subseteq I_{\sigma}$ and $1 \in I_{\sigma}$ are incomparable with respect to $\leq_{S^{1}}$ : if $\xi<_{S^{1}} 1$ then $\xi \in E_{S}$ (by [8], Lemma 2.1), a contradiction; $1<_{S^{1}} \xi$ is impossible by Lemma 3.1, below. Therefore, the $\sigma$-class $I_{\sigma}$ of $S^{1}$ has no greatest element, a contradiction.

Sufficiency. Let $\rho$ be the corresponding group congruence on $S$. By [2], Corollary 3.9, the identity $\rho$-class $I$ of $S$ is either $E_{S}$ or $E_{S} \cup\{a\}$ with $a \notin E_{S}$, the greatest element of $I$. By hypothesis, the pivot $\xi$ of $S$ is idempotent, whence $I=E_{S}$. Let $\sigma$ be the equivalence relation on $S^{1}$ given by the partition $\rho$ on $S$ but with $1 \sigma=I \cup\{1\}$. Then $\sigma$ is a congruence on $S^{1}$. Only the case $e \sigma 1$, $x \in S, e \in E_{S}=I$, has to be considered: in the group $S / \rho, I=e \rho$ is the identity element hence $(e x) \rho=(e \rho)(x \rho)=x \rho$ and ex $\rho x$, thus also ex $\sigma x$; similarly $x e \sigma x$. Evidently, $S^{1} / \sigma$ is a group, whose identity element $1 \sigma=E_{S} \cup\{1\}=E_{S^{1}}$ has $1 \in S^{1}$ as greatest element. All the other $\sigma$-classes of $S^{1}$ are equal to the $\rho$ classes of $S$, thus admit each a greatest element with respect to $\leq_{S}$, whence also with respect to $\leq_{S^{1}}$. Therefore, $S^{1}$ is an $F$-monoid.

This result allows the construction of further examples of $F$-monoids. Let $T$ be a semigroup; for every $\alpha \in T$ let $T_{\alpha}$ be any set with $T_{\alpha} \cap T=\{\alpha\}$ and $T_{\alpha} \cap T_{\beta}=\emptyset$ for all $\alpha \neq \beta$. Then $S=\underset{\alpha \in T}{\cup} T_{\alpha}$ forms a semigroup with respect to the operation

$$
a \cdot b=\alpha \beta \text { if } a \in T_{\alpha}, b \in T_{\beta},
$$

called an inflation of $T$ (see [11]). $S$ is a proper inflation of $T$ if $T_{\alpha} \neq\{\alpha\}$ for at least one $\alpha \in T$. Note that a proper inflation $S$ of $T$ can not have an identity since for $a \in T_{\alpha}, a \neq \alpha$, we would have $a 1_{S} \in T$, but $a \notin T$. Also $S$ is not 
regular, since $a x a \in T$ for any $x \in S$ - but $a \notin T$. Finally, $E_{S}=E_{T}$ since $x^{2} \in T$ for any $x \in S$. Specializing $T$ we obtain

(5) Let $T=G$ be a group and $S=\underset{g \in G}{\cup} T_{g}$ be a proper inflation of $G$, such that $\left|T_{g}\right| \leq 2$ for every $g \in G, g \neq 1_{G}$, and $T_{1_{G}}=\left\{1_{G}\right\}$. Then by [3], Corollary $6.2, S$ is an $F$-semigroup with pivot $\xi=1_{G} \in E_{S}$ (note that $1_{G}$ is the unique idempotent of $S$ and that $1_{G}$ is maximal in $\left(S, \leq_{S}\right)$ : see Lemma 3.1, below). It follows by Proposition 2.1, that $S^{1}$ is an $F$-monoid (with pivot $\xi=1$ ). Note that $\leq_{S}$ is compatible with multiplication since $\leq_{G}=i d_{G}$ is so (see [8]).

More generally we have

(6) Let $T$ be an $F$-semigroup such that for every $\alpha \in T$ there exist $\beta, \gamma \in T$ with $\alpha=\beta \alpha=\alpha \gamma$, and with pivot $\xi \in E_{T}$ (the greatest idempotent of $T$ ). Let $S=\underset{\alpha \in T}{\cup} T_{\alpha}$ be a proper inflation of $T$ such that $\left|T_{\mu}\right| \leq 2$ for every maximal $\mu \in T$ and $\left|T_{\alpha}\right|=\left|T_{\xi}\right|=1$ for every non-maximal $\alpha \in T$. Then by [3], Theorem $6.1, S$ is an $F$-semigroup with pivot $\xi \in E_{T}$. It follows by Proposition 2.1, that $S^{1}$ is an $F$-monoid (with pivot $\xi=1$ ). Examples for $T$ are: groups (see Example (5)); bands with identity, more generally $F$-regular semigroups (see [4]) - in any of these cases, $\xi=1_{T} \in E_{T}$. Note that $\leq_{S}$ is compatible with multiplication if and only if $\leq_{T}$ is so (see [8]).

\section{Characterizations}

A general theory of $F$-semigroups was developed in [3]. Specializing to the case that an identity exists, we obtain the following characterizations of $F$-monoids. First we give a direct proof of a useful result on the pivot.

Lemma 3.1 If $S$ is a monoid then $1_{S}$ is a maximal element in $\left(S, \leq_{S}\right)$. In particular, if $S$ is a generalized $F$-monoid then the pivot $\xi$ of $S$ is $1_{S}$.

Proof. If $1_{S} \leq_{S} a$ for some $a \in S$ then $1_{S}=x a=x 1_{S}=x$ for some $x \in S$, hence $1_{S}=a$.

If $S$ is a generalized $F$-monoid with pivot $\xi$ then the identity $\rho$-class $I$ of $S$ is of the form $I=(\xi]$. Since $1_{S} \in E_{S}$ and $\rho$ is a group congruence, it follows that $1_{S} \in I$, whence $1_{S} \leq_{S} \xi$ and $1_{S}=\xi$.

We begin with the more general situation of generalized $F$-monoids (see [2], Corollary 3.12).

Proposition 3.2 Let $S$ be a monoid. Then $S$ is a generalized $F$-semigroup if and only if $S$ is E-inversive and E-unitary.

The first characterization of $F$-monoids was given in [3], Theorem 4.5 , describing them as particular $E$-inversive semigroups in terms of the natural partial order:

Theorem 3.3 Let $S$ be a monoid. Then $S$ is an F-monoid if and only if for every $a \in S$ there exists a greatest element $x \in S$ (with respect to $\leq_{S}$ ) such that $a x \in E_{S}$. 
Note that by [11], Exercise I.7(14), a semigroup $S$ such that for any $a \in S$ there is a unique $x \in S$ with $a x \in E_{S}$, is a group (hence an $F$-monoid - see [3]). The second characterization is tightly connected with that of Theorem 3.3:

Theorem 3.4 Let $S$ be a monoid. Then $S$ is an F-monoid if and only if the identity $1_{S} \in S$ is right (left; equi) residuated, i.e., for every a $\in S$, $\max \left\{x \in S \mid a x \leq_{S} 1_{S}\right\}=1_{S} \cdot{ }^{\prime}$ a exists $\left(\max \left\{x \in S \mid x a \leq_{S} 1_{S}\right\}=1_{S} \cdot\right.$ a exists; both exist and are equal: $\left.1_{S} \cdot a=1_{S} \cdot a\right)$.

Proof. Necessity. By Lemma 3.1, $\xi=1_{S}$. Thus the statement follows from [3], Theorem 3.5.

Sufficiency. Let $a \in S$ and let $x_{0} \in S$ be the greatest element of all $x \in S$ such that $a x \leq_{S} 1_{S}$. Since by [8], Lemma 2.1:

$$
\left\{x \in S \mid a x \leq_{S} 1_{S}\right\}=\left\{x \in S \mid a x \in E_{S}\right\},
$$

$x_{0} \in S$ is the greatest element in $S$ such that $a x_{0} \in E_{S}$. It follows by Theorem 3.3 , that $S$ is an $F$-semigroup.

The third characterization uses the sets $T(a)=\left\{x \in S \mid a x a \leq_{S} a\right\}, a \in S$. By means of these sets, first we provide a description of the $\rho$-classes of an $F$-monoid, more generally of an $F$-semigroup with regular pivot.

Proposition 3.5 Let $S$ be an F-semigroup with regular pivot $\xi$. Then for any $a \in S,(a \rho)^{-1}=T(a)$.

Proof. Let $a \in S$. If $x \in T(a)$, then $a x a \leq_{S} a$. Applying the natural homomorphism of $S$ onto $G=S / \rho$ we obtain $(a x a) \rho=a \rho$. Thus $(a \rho)(x \rho)(a \rho)=a \rho$ so that by cancellation in $G,(a \rho)(x \rho)=1_{G}$ and $x \rho=(a \rho)^{-1}$, i.e., $x \in(a \rho)^{-1}$. Conversely, let $x \in(a \rho)^{-1}$. Then $x \rho=(a \rho)^{-1}$ and $(a x) \rho=(a \rho)(x \rho)=1_{G}=$ $(\xi]$, i.e., $a x \leq_{S} \xi$. Since $\xi \in S$ is regular, $\xi \in E_{S}$ by [2], Proposition 3.13. It follows by [8], Lemma 2.1, that $a x \in E_{S}$ too. Hence $a x a=a x \cdot a=a \cdot x a$ implies that $\operatorname{axa} \leq_{S}$ a, i.e., $x \in T(a)$.

Remark. There are non-regular $F$-semigroups with regular pivot - see Example (5) in Section 2.

Since by Lemma 3.1, for an $F$-monoid $S$ the pivot $\xi=1_{S}$ is regular we obtain

Corollary 3.6 Let $S$ be an F-monoid. Then for any $a \in S,(a \rho)^{-1}=T(a)$ and $\max T(a)$ exists in $\left(S, \leq_{S}\right)$.

We will show now that this last property of a monoid $S$ is also sufficient for $S$ to be an $F$-semigroup. By Theorem 3.3 in [4], a generalized $F$-semigroup $S$ with regular pivot is an $F$-semigroup if and only if $\max T(a)$ exists for any $a \in S$. In order to apply Proposition 3.2 , which describes generalized $F$-monoids, we first give a new characterization of $E$-inversive semigroups. 
Lemma 3.7 A semigroup $S$ is E-inversive if and only if for any a $\in S$ there exists $x \in S$ such that axa $\leq_{S}$ a (i.e., if and only if $\left.T(a) \neq \emptyset\right)$.

Proof. If $S$ is $E$-inversive then for any $a \in S$ there is some $x \in S$ such that $a x \in E_{S}$. Therefore, $a x a=a x \cdot a=a \cdot x a$ implies that $a x a \leq_{S} a$. Conversely, let $a \in S, x \in S$ be such that $a x a \leq_{S} a$. If $a x a=a$ then $a x \in E_{S}$. If $a x a<_{S} a$ then $a x a=y \cdot a=a \cdot z, y \cdot a x a=a x a$, for some $y, z \in S$. Hence

$$
(a x)^{3}=a x a \cdot x a x=y a \cdot x a x=y a x a \cdot x=a x a \cdot x=(a x)^{2}
$$

and $(a x)^{4}=(a x)^{2} \in E_{S}$. Thus $a \cdot x a x \in E_{S}$, i.e., $S$ is $E$-inversive.

Theorem 3.8 Let $S$ be a monoid. Then $S$ is an F-monoid if and only if for any $a \in S, \max T(a)$ exists in $\left(S, \leq_{S}\right)$.

Proof. Necessity holds by Corollary 3.6.

Sufficiency. First, by Lemma 3.7, $S$ is $E$-inversive. Next, we show that $S$ is $E$-unitary. Let $e, e x \in E_{S}$. Then $e x e=e x \cdot e=e \cdot x e$ implies that $e x e \leq_{S} e$, i.e., $x \in T(e)$. Since $e 1_{S} e=e \leq_{S} e$, we have $1_{S} \in T(e)$ and $1_{S} \leq_{S} \max T(e)$. It follows by Lemma 3.1, that $1_{S}=\max T(e)$. Therefore, $x \leq_{S} 1_{S}$, so that by [8], Lemma 2.1, $x \in E_{S}$. Thus, by Proposition 3.2, $S$ is a generalized $F$-monoid. Hence, by Lemma 3.1, the pivot of $S$ is $\xi=1_{S}$, i.e., $\xi$ is regular. Therefore by [4], Theorem 3.3, $S$ is an $F$-semigroup.

Remark. In the language of partially ordered semigroups, Theorem 3.8 says that a monoid $S$ is principally ordered with respect to its natural partial order $\leq_{S}$ (see [4]) if and only if $S$ is an $F$-monoid. Notice that $\leq_{S}$ is not compatible with multiplication, in general (see [8]) - but note Examples (2) (6) in Section 2.

The next characterization of $F$-monoids $S$ is in terms of the maximal elements in $\left(S, \leq_{S}\right)$ :

Theorem 3.9 Let $S$ be a monoid. Then $S$ is an F-monoid if and only if

(i) $S$ is E-inversive;

(ii) for every $a \in S$, there exists a unique maximal $m \in S$ such that $a \leq_{S} m$;

(iii) if $a, b \in S$ are included in the same maximal element then so are $a c, b c$ resp. $c a, c b$, for any $c \in S$.

Proof. Necessity holds by [3], Theorem 5.3.

Sufficiency. Let $T=\left\{m_{i} \mid i \in I\right\}$ be the set of all maximal elements of $\left(S, \leq_{S}\right)$. By (ii), $T \neq \emptyset$ and $S$ is the disjoint union of the principal order ideals $\left(m_{i}\right](i \in I)$ in $\left(S, \leq_{S}\right)$. Define

$$
a \rho b \Leftrightarrow a, b \in\left(m_{i}\right] \text { for some } i \in I \text {. }
$$


Using (ii) and (iii) it is easy to show that $\rho$ is a congruence on $S$. Thus $S / \rho$ is a semigroup with $1_{S} \rho$ as identity element. If $e \in E_{S}$ then $e \leq_{S} 1_{S}$ and $e, 1_{S} \in\left(1_{S}\right]$. Since by Lemma $3.1,1_{S}$ is a maximal element in $\left(S, \leq_{S}\right)$, it follows that $e \rho 1_{S}$. Let $a \rho \in S / \rho$; then by (i), $a x=f \in E_{S}$ for some $x \in S$. Thus, $(a \rho)(x \rho)=(a x) \rho=f \rho=1_{S} \rho$; therefore $S / \rho$ is a group. Furthermore, if $a \in\left(m_{i}\right]$ for $i \in I$, say, then we have that $a \rho=\left(m_{i}\right]$. Hence $S$ is an $F$-semigroup.

By [3], Corollary 5.6, we have

Corollary 3.10 Let $S$ be a monoid with compatible natural partial order. Then $S$ is an F-monoid if and only if $S$ is E-inversive and for every $a \in S$ there exists a unique maximal $m \in S$ such that $a \leq_{S} m$.

Remark. Examples of semigroups $S$ with compatible natural partial order are: commutative or centric (i.e., $a S=S a$ for every $a \in S$ ) or inverse semigroups (see [8]). Note that the latter are also $E$-inversive.

Following an idea of M. Petrich (see [3], Theorem 3.9, on $F$-semigroups) we obtain an axiomatic description of $F$-monoids by means of an additional unary operation with certain properties reflecting those of the greatest elements in the different $\rho$-classes.

Theorem 3.11 Let $S$ be a monoid. Then $S$ is an F-monoid if and only if $S$ has a unary operation $a \rightarrow a^{\prime}$ satisfying

(F1) $(a b)^{\prime}=\left(a^{\prime} b\right)^{\prime}=\left(a b^{\prime}\right)^{\prime}$ for all $a, b \in S$;

(F2) for every $a \in S, a \leq_{S} a^{\prime}$;

(F3) for any $a \in S$ there exists $b \in S$ such that $(a b)^{\prime}=1_{S}$.

Proof. Necessity. Let $\rho$ be the defining group congruence on $S$ and for any $a \in S$, let $a^{\prime}$ be the greatest element of the $\rho$-class $a \rho \in S / \rho$. Then by [3], Theorem 3.9, (F1) and (F2) hold. In particular, $1_{S} \leq_{S} 1_{S}^{\prime}$; hence $1_{S}=1_{S}^{\prime}$ (by Lemma 3.1). Let $a \in S$; since $S / \rho$ is a group there exists $b \rho \in S / \rho$ such that $(a \rho)(b \rho)=1_{S} \rho$ (the identity of $\left.S / \rho\right)$. Hence $(a b) \rho=1_{S} \rho$ and $(a b)^{\prime}=1_{S}^{\prime}=1_{S}$.

Sufficiency. Define a relation $\rho$ on $S$ by: $a \rho b \Leftrightarrow a^{\prime}=b^{\prime}$. Then by (F1), $\rho$ is a congruence on $S$. Evidently, $1_{S} \rho$ is the identity of the semigroup $S / \rho$. Note that by (F2), $1_{S} \leq_{S} 1_{S}^{\prime}$, whence $1_{S}=1_{S}^{\prime}$ (by Lemma 3.1). Let $a \rho \in S / \rho$; then by (F3) there exists $b \in S$ such that $(a b)^{\prime}=1_{S}=1_{S}^{\prime}$. It follows that $a b \rho 1_{S}$, so that in $S / \rho$ we have $(a \rho)(b \rho)=1_{S} \rho$. Therefore, $S / \rho$ is a group. Let $a \in S$; then $a^{\prime} \in S$ is the greatest element of the $\rho$-class $a \rho \in S / \rho$ by definition of $\rho$ and (F2) (see [3], Theorem 3.9). It follows that $S$ is an $F$-semigroup.

Corollary 3.12 Let $S$ be a monoid. Then $S$ is an F-monoid if and only if $S$ is E-inversive and has a unary operation $a \rightarrow a^{\prime}$ satisfying

(F1) $(a b)^{\prime}=\left(a^{\prime} b\right)^{\prime}=\left(a b^{\prime}\right)^{\prime}$ for all $a, b \in S$;

(F2) for every $a \in S, a \leq_{S} a^{\prime}$;

(F4) for any $e \in E_{S}, e^{\prime}=1_{S}$. 
Proof. Necessity. First, by Proposition 3.2, $S$ is $E$-inversive. Let $\rho$ be the defining group congruence on $S$ and for any $a \in S$, let $a^{\prime}$ denote the greatest element of $a \rho \in S / \rho$. Then (F1) and (F2) hold by Theorem 3.11; in particular, $1_{S}^{\prime}=1_{S}$ (by Lemma 3.1). Let $e \in E_{S}$; then $e \rho 1_{S}$ and $e^{\prime}=1_{S}^{\prime}=1_{S}$.

Sufficiency. Let $a \in S$; then $a x \in E_{S}$ for some $x \in S$ (since $S$ is $E$-inversive). It follows by (F4), that $(a x)^{\prime}=1_{S}$. Consequently, $S$ is an $F$-semigroup (by Theorem 3.11).

\section{Three unary operations}

Let $S$ be an $F$-monoid and $\rho$ the corresponding group congruence on $S$. Then by Lemma 3.1, the pivot of $S$ is $\xi=1_{S}$ and by Theorem 3.4, for any $a \in S$, $1_{S}: a=\max \left\{x \in S \mid a x \leq_{S} 1_{S}\right\}=\max \left\{x \in S \mid x a \leq_{S} 1_{S}\right\}$ exists in $\left(S, \leq_{S}\right)$.

Since $a\left(1_{S}: a\right) \leq_{S} 1_{S}$ and $\left(1_{S}: a\right) a \leq_{S} 1_{S}$, it follows by [8], Lemma 2.1, that $a\left(1_{S}: a\right),\left(1_{S}: a\right) a \in E_{S}$. Furthermore, by definition, each $\rho$-class $a \rho$ of $S$ has a greatest element. Recall from [3], Corollary 3.3, that the greatest element of $(a \rho)^{-1} \in S / \rho$ is $1_{S}: a \in S$ and that of $a \rho \in S / \rho$ is $1_{S}:\left(1_{S}: a\right)$.

As the first unary operation $a \rightarrow a^{*}$ on $S$ we define

$$
a^{*}=1_{S}: a \text { for any } a \in S(\text { see }[4]) .
$$

Hence, $a^{*} \in S$ is the greatest element of $(a \rho)^{-1} \in S / \rho$, and $\left(a^{*}\right)^{*}=a^{* *} \in S$ is that of $a \rho \in S / \rho$. Then in the notation in the proof of Theorem 3.11, $a^{\prime}=a^{* *}$. With this observation in mind, Theorem 3.11 and Corollary 3.12 remain true if the unary operation is given by $a \rightarrow a^{* *}$ and in conditions (F1), (F2), (F3) and (F4) the symbol' is replaced by ${ }^{* *}$.

Since $a a^{*}, a^{*} a \in E_{S}, a a^{*} a=a a^{*} \cdot a=a \cdot a^{*} a$ implies that $a a^{*} a \leq_{S} a$. Furthermore, by [4], Theorem 3.3 and its proof, $a^{*} \in S$ is the greatest element of all $x \in S$ such that $a x a \leq_{S} a$. Since $a a^{*} a \cdot a^{*} \cdot a a^{*} a=\left(a a^{*}\right)^{3} a=a a^{*} a$, the element $a a^{*} a \in S$ is regular.

We define our second unary operation $a \rightarrow \bar{a}$ on $S$ by

$$
\bar{a}=a a^{*} a \text { for any } a \in S \text {. }
$$

Lemma 4.1 Let $S$ be an F-monoid. If $R(S)$ denotes the set of all regular elements of $S$ then $R(S)=\{\bar{a} \mid a \in S\}$ and $R(S)$ is a regular subsemigroup of $S$.

Proof. By [4], Theorem 2.2, every regular element $a \in S$ satisfies $a=$ $a\left(1_{S}: a\right) a=a a^{*} a=\bar{a}$. Thus $R(S) \subseteq\{\bar{a} \mid a \in S\}$. The converse inclusion was shown above. Also, by [2], Proposition 3.7, $E_{S}$ forms a subsemigroup of $S$. Hence the second assertion follows by [5] (see also [10], Lemma 5.2).

In the following we collect several properties of the operations $*$ and - . Recall that $A(a)=\{x \in S \mid a x a=a\}$ is the set of associates of $a \in S$. 
Proposition 4.2 Let $S$ be an F-monoid. Then for all $a, b \in S, e \in E_{S}$ the following hold:

(i) $a \leq_{S} a^{* *}$;

(ii) $e^{*}=1_{S}$;

(iii) $a \leq_{S} b \Rightarrow a^{*}=b^{*}, a a^{*} \leq_{S} b b^{*}, a^{*} a \leq_{S} b^{*} b$;

(iv) $a^{*}=a^{* * *}$;

(v) $a a^{*} \leq_{S} a^{* *} a^{*}, a^{*} a \leq_{S} a^{*} a^{* *} ;$

(vi) $(e a)^{*}=a^{*}=(a e)^{*}$;

(vii) If $a \in S$ is regular then $a^{\prime} \rho a^{*}$ for all $a^{\prime} \in A(a)$;

(viii) $a^{*}=\bar{a}^{*}$;

(ix) $a^{* *}=\max A\left(\overline{a^{*}}\right)$.

Proof. (i), (ii), (iv), (v), (vi) and (vii) are proved as in [4], Proposition 4.1. (iii) Let $a \leq_{S} b$; then by the proof of [4], Proposition 4.1 (ii), $a^{*}=b^{*}$. Next, $\bar{a}=a^{*} a a^{*} \leq_{S} a \leq_{S} b$ implies by [8] Lemma 2.1, that $\bar{a}=e b=b f$ for some $e, f \in E_{S}$. Hence

$$
a a^{*}=a a^{*} a \cdot a^{*}=\bar{a} \cdot a^{*}=e b \cdot a^{*}=e \cdot b b^{*}, a a^{*}=b f b^{*} .
$$

Now by [3], Lemma 3.7, and by (ii), $1_{S}: f b^{*}=\left(1_{S}: f\right): b^{*}=1_{S}: b^{*}$. Thus we obtain from [3], Corollary 3.4, that $f b^{*} \rho b^{*}$. Therefore $f b^{*} \leq_{S} b^{*}(=$ the greatest element of its $\rho$-class). It follows that $f b^{*}=b^{*} x$ for some $x \in S$. Thus $a a^{*}=b f b^{*}=b b^{*} x$ and $a a^{*} \leq_{S} b b^{*}$. Similarly, $a^{*} a \leq_{S} b^{*} b$.

(viii) Since $a a^{*} \in E_{S}$ we have by (vi), that $\bar{a}^{*}=\left(a a^{*} a\right)^{*}=a^{*}$.

(ix) By Lemma 4.1, $\overline{a^{*}}$ is regular. Hence by [4], Corollary 2.3 (with $\xi=1_{S}$ )

$$
\max A\left(\overline{a^{*}}\right)=\left(\overline{a^{*}}\right)^{*}=\left(a^{*} a^{* *} a^{*}\right)^{*}=a^{* *},
$$

where the last equality holds by (vi), since $a^{*} a^{* *} \in E_{S}$.

Our third unary operation $a \rightarrow a^{\circ}$ on $S$ is defined by

$$
a^{\circ}=a^{*} a a^{*}, \text { where } a^{*}=1_{S}: a(\text { see }[4]) .
$$

Since $a a^{*}, a^{*} a \in E_{S}$, also $a a^{\circ}, a^{\circ} a \in E_{S}$. Recall that $V(a)=\{x \in S \mid a=a x a, x=x a x\}$.

Proposition 4.3 Let $S$ be an F-monoid. Then we have for all $a, b \in S, e \in E_{S}$ :

(i) $a^{\circ} \in V(\bar{a})$ and $a \in A\left(a^{\circ}\right)$;

(ii) $a a^{\circ}=a a^{*}=\bar{a} a^{\circ}$ and $a^{\circ} a=a^{*} a=a^{\circ} \bar{a}$;

(iii) if $a^{\prime} \in V(\bar{a}), a^{\prime} \neq a^{\circ}$, then $a^{\circ}$ is incomparable with $a^{\prime}$; 
(iv) $a^{* \circ} \leq_{S} a^{* *}$ and $a^{\circ *}=a^{* *}$;

(v) $e^{\circ}=e$;

(vi) $a a^{*}=a^{* *} a^{\circ}$ and $a^{*} a=a^{\circ} a^{* *}$;

(vii) $a^{\circ \circ}=\bar{a}$ and $a^{\circ \circ} \leq_{S} a$;

(viii) $a^{\circ 00}=a^{\circ}$;

(ix) $a^{* *} a^{*} a=\bar{a}=a a^{*} a^{* *}$;

(x) $a \leq_{S} b \Rightarrow a^{\circ} \leq_{S} b^{\circ}, a a^{\circ} \leq_{S} b b^{\circ}, a^{\circ} a \leq_{S} b^{\circ} b$;

(xi) $\overline{a^{\circ}}=a^{\circ}=\bar{a}^{\circ}$.

Proof. (i) $a^{\circ} \bar{a} a^{\circ}=a^{*} a a^{*} \cdot a a^{*} a \cdot a^{*} a a^{*}=\left(a^{*} a\right)^{4} a^{*}=a^{*} a a^{*}=a^{\circ}$, $\bar{a} a^{\circ} \bar{a}=a a^{*} a \cdot a^{*} a a^{*} \cdot a a^{*} a=\left(a a^{*}\right)^{4} a=a a^{*} a=\bar{a} ;$

$a^{\circ} a a^{\circ}=a^{*} a a^{*} \cdot a \cdot a^{*} a a^{*}=\left(a^{*} a\right)^{3} a^{*}=a^{*} a a^{*}=a^{\circ}$.

(ii) $a a^{\circ}=a \cdot a^{*} a a^{*}=a a^{*} ; \bar{a} a^{\circ}=a a^{*} a \cdot a^{*} a a^{*}=\left(a a^{*}\right)^{3}=a a^{*}$. Similarly we prove the other equalities.

(iii) Let $a^{\prime} \in V(\bar{a})$ be such that $a^{\prime} \neq a^{\circ}$ (see (i)), and assume that $a^{\prime}<_{S} a^{\circ}$. Then since $a^{\prime} \in S$ is regular we have by [8], Lemma 2.1, that $a^{\prime}=e a^{\circ}=a^{\circ} f$ for some $e, f \in E_{S}$. Thus, by (i),

$$
\begin{aligned}
& \bar{a} a^{\prime}=\bar{a} \cdot e a^{\circ}=\bar{a} e \cdot a^{\circ} \bar{a} a^{\circ}=\bar{a} a^{\prime} \bar{a} \cdot a^{\circ}=\bar{a} a^{\circ}, \\
& a^{\prime} \bar{a}=a^{\circ} f \cdot \bar{a}=a^{\circ} \bar{a} a^{\circ} \cdot f \bar{a}=a^{\circ} \cdot \bar{a} a^{\prime} \bar{a}=a^{\circ} \bar{a} .
\end{aligned}
$$

Hence, $a^{\prime}=a^{\prime} \bar{a} a^{\prime}=a^{\circ} \bar{a} \cdot a^{\prime}=a^{\circ} \cdot \bar{a} a^{\circ}=a^{\circ}$ (by (i)): contradiction. The proof for $a^{\circ}<_{S} a^{\prime}$ is obtained by interchanging $a^{\circ}$ and $a^{\prime}$.

(iv) $a^{\circ *}=\left(a^{*} a a^{*}\right)^{*}=a^{* *}$, by Proposition $4.2\left(\right.$ vi) (since $a^{*} a \in E_{S}$ ); $a^{* 0}=a^{* *} a^{*} a^{* *} \leq_{S} a^{* *}\left(\right.$ since $\left.a^{* *} a^{*}, a^{*} a^{* *} \in E_{S}\right)$.

(v) and (vi) are proved as in [4], Proposition 4.2.

(vii)

$$
\begin{aligned}
a^{\circ \circ} & =a^{\circ *} a^{\circ} a^{\circ *} & & (\text { by (iv) }) \\
& =a^{* *} a^{\circ} a^{* *} & & \\
& =a^{* *} \cdot a^{\circ} \bar{a} a^{\circ} \cdot a^{* *} & & \text { by (i) }) \\
& =a a^{*} \cdot \bar{a} \cdot a^{*} a & & \text { (by (vi)) } \\
& =a a^{*} \cdot a a^{*} a \cdot a^{*} a & & \\
& =a a^{*} a & & \\
& =\bar{a} \leq_{S} a . & & \text { (see the beginning of this Section) }
\end{aligned}
$$

(viii)

$$
\begin{aligned}
& a^{\circ 00}=\left(a^{\circ 0}\right)^{\circ} \\
& =(\bar{a})^{\circ} \\
& =\left(a a^{*} a\right)^{\circ} \\
& =\left(a a^{*} a\right)^{*} a a^{*} a\left(a a^{*} a\right)^{*} \\
& =a^{*} \cdot a a^{*} a \cdot a^{*} \\
& =a^{*} a a^{*} \\
& =a^{\circ} \text {. }
\end{aligned}
$$


(ix) By (vii), (iv) and (vi), $\bar{a}=a^{\circ \circ}=a^{\circ *} a^{\circ} a^{\circ *}=a^{* *} \cdot a^{\circ} a^{* *}=a^{* *} \cdot a^{*} a$. Similarly $a a^{*} a^{* *}=\bar{a}$.

(x) Let $a \leq_{S} b$; then by Proposition 4.2 (iii), $a^{*}=b^{*}$ and $a a^{*} \leq_{S} b b^{*}$. It follows by (ii), that $a a^{\circ} \leq_{S} b b^{\circ}$ and similarly $a^{\circ} a \leq_{S} b^{\circ} b$. Furthermore, since $a a^{*}, b b^{*} \in E_{S}$ we have $a a^{*}=b b^{*} \cdot a a^{*}=a a^{*} \cdot b b^{*}$. Therefore

$$
\begin{gathered}
a^{\circ}=a^{*} \cdot a a^{*}=b^{*} \cdot b b^{*} a a^{*}=b^{\circ} \cdot a a^{*}, \\
a^{\circ}=a^{*} \cdot a a^{*}=a^{*} \cdot a a^{*} b b^{*}=a^{*} a \cdot b^{*} b b^{*}=a^{*} a \cdot b^{\circ},
\end{gathered}
$$

i.e., $a^{\circ} \leq_{S} b^{\circ}$.

(xi) Since by (i) $a^{\circ} \in R(S)$, it is obvious by [4], Theorem 2.2, that $a^{\circ}=\overline{a^{\circ}}$. Also by Proposition 4.2 (viii) and $a^{*} a \in E_{S}$ we have $\bar{a}^{\circ}=\bar{a}^{*} \overline{a a}^{*}=a^{*} a a^{*} a a^{*}=$ $a^{*} a a^{*}=a^{\circ}$.

Remark. The inequality in (iv) may be strict. Consider the non-regular $F$-monoid in Example (3) of Section 2. The corresponding group congruence on $S$ has the classes: $\{-\bar{n}\}_{n \in \mathbb{N}},\{n, \bar{n}\}_{n \in \mathbb{N}_{0}}$. The greatest elements of these classes are: $-\bar{n}(n \in \mathbb{N})$ and $n\left(n \in \mathbb{N}_{0}\right)$ - note that $\bar{n}=n \varphi_{\omega, \mu}$ implies that $\bar{n}<_{S} n$. Hence we have for every $a \in S: a^{* *}=-\bar{n}$ if $a=-\bar{n}$, and $a^{* *}=n$ if $a \in\{n, \bar{n}\}$. Thus for the non-regular element $a=n \in \mathbb{N} \subseteq S_{\omega}$ we obtain that $a^{* *}=n$; but $a^{* \circ}=\left(a^{*}\right)^{\circ}=(-\bar{n})^{\circ}=-\bar{n}+n+(-\bar{n})=-\bar{n}+\bar{n}-\bar{n}=-\bar{n}$, i.e, $a^{* \circ} \neq a^{* *}$.

In fact we have the following general result:

Lemma 4.4 Let $S$ be an F-monoid. Then for any $a \in S, a^{* \circ}=a^{* *}$ if and only if $S$ is regular.

Proof. Necessity. Let $a \in S$; then $a^{* *}=a^{* \circ}=a^{* *} a^{*} a^{* *}$ and $a^{* *} \in S$ is regular. That is, the greatest element of the $\rho$-class $a \rho \in S / \rho$ is regular. From $a \leq_{S} a^{* *}$ it follows by [8], Lemma 2.1, that $a \in S$ is regular, too.

Sufficiency. Let $a \in S$; then since $a^{* *} \in S$ is regular, $a^{* *}=a^{* *} a^{* * *} a^{* *}$ by [4], Corollary 2.4. By Proposition 4.2 (iv), $a^{*}=a^{* * *}$. Thus it follows that $a^{* \circ}=a^{* *} a^{*} a^{* *}=a^{* *} a^{* * *} a^{* *}=a^{* *}$.

The operation $a \rightarrow a^{\circ}$ on an $F$-monoid $S$ gives rise to the subsemigroup

$$
S^{\circ}=\left\{a^{\circ} \in S \mid a \in S\right\}
$$

of $S$. This semigroup will play an important role in the following section where a further characterization of $F$-monoids will be given. As a first step we show

Proposition 4.5 Let $S$ be an F-monoid. Then $S^{\circ}=R(S)$ and $S^{\circ}$ is a regular subsemigroup of $S$.

Proof. Let $a^{\circ} \in S^{\circ}$; then by Proposition 4.3 (i), $a^{\circ}$ is regular, hence $S^{\circ} \subseteq$ $R(S)$. Conversely, $R(S)=\{\bar{a} \mid a \in S\}$ by Lemma 4.1. But by Proposition 4.3 (vii), $\bar{a}=a^{\circ \circ}$ for any $a \in S$. Therefore $R(S) \subseteq S^{\circ}$, and equality prevails. The second assertion holds by Lemma 4.1 .

Remark. Notice that Lemma 4.1 together with Proposition 4.5 give that $R(S)=S^{\circ}=\{\bar{a}: a \in S\}$. 


\section{A characterization by the regular part}

In this Section we give a description of $F$-monoids $S$ by means of the regular part $R(S)=S^{\circ}$ of $S$ (see Proposition 4.5). It turns out that $S^{\circ}$ is an $F$ regular semigroup, the structure of which was studied in [4]. Furthermore, the non-regular elements of $S$ are associates of elements in $S^{\circ}$ as we will show first.

Lemma 5.1 Let $S$ be an F-monoid; then $S=A\left(S^{\circ}\right)$.

Proof. Let $a \in S$; then by Proposition 4.3 (i), $a \in A\left(a^{\circ}\right)$. Hence $S \subseteq A\left(S^{\circ}\right)$ and equality prevails.

Proposition 5.2 Let $S$ be an F-monoid. Then $S^{\circ}$ is an F-regular subsemigroup of $S$.

Proof. By Proposition 4.5, $S^{\circ}=R(S)$ is a regular, hence $E$-inversive subsemigroup of $S$. We will define a unary operation $a^{\circ} \rightarrow\left(a^{\circ}\right)^{\prime}$ on $S^{\circ}$ satisfying (F1), (F2) and (F4) of Corollary 3.12. Then it follows that $S^{\circ}$ is an $F$-regular monoid (note that $1_{S} \in S^{\circ}$ ). Consider the operation $a^{\circ} \rightarrow\left(a^{\circ}\right)^{\prime}=\left(a^{\circ}\right)^{* \circ}$ on $S^{\circ}$.

Since $a^{*} a \in E_{S}$ we first have by Proposition 4.2 (vi):

$$
\left(a^{\circ}\right)^{\prime}=\left(a^{*} a a^{*}\right)^{* \circ}=a^{* * \circ} .
$$

(F1) For any $a^{\circ}, b^{\circ} \in S^{\circ}$ :

$$
\left(a^{\circ} b^{\circ}\right)^{\prime}=\left(a^{\circ} b^{\circ}\right)^{* \circ}=\left(a^{*} a a^{*} \cdot b^{*} b b^{*}\right)^{* \circ}=\left(a^{*} a \cdot a^{*} b^{*} \cdot b b^{*}\right)^{* \circ}=\left(a^{*} b^{*}\right)^{* \circ}
$$

by Proposition $4.2(\mathrm{vi})$, since $a^{*} a, b b^{*} \in E_{S}$; on the other hand

$$
\left.\left(a^{\circ}\right)^{\prime}=\left(a^{* *}\right)^{\circ}=a^{* * *} a^{* *} a^{* * *}=a^{*} a^{* *} a^{*} \text { (by Proposition } 4.2(\mathrm{iv})\right)
$$

and thus

$$
\left[\left(a^{\circ}\right)^{\prime} b^{\circ}\right]^{\prime}=\left(a^{*} a^{* *} a^{*} \cdot b^{*} b b^{*}\right)^{* \circ}=\left(a^{*} a^{* *} \cdot a^{*} b^{*} \cdot b b^{*}\right)^{* \circ}=\left(a^{*} b^{*}\right)^{* \circ}
$$

by Proposition $4.2(\mathrm{vi})$, since $a^{*} a^{* *}, b b^{*} \in E_{S}$.

Similarly, $\left[a^{\circ}\left(b^{\circ}\right)^{\prime}\right]^{\prime}=\left(a^{*} b^{*}\right)^{* \circ}$.

(F2) For any $a \in S$ by Proposition 4.2 (i), $a \leq_{S} a^{* *}$; hence $a^{\circ} \leq_{S} a^{* * \circ}=$ $\left(a^{\circ}\right)^{\prime}$ by Proposition $4.3(\mathrm{x})$.

(F4) For any $e \in E_{S} \subseteq S^{\circ}$ we have by Proposition $4.3(\mathrm{v}), e=e^{\circ}$; hence $e^{\prime}=\left(e^{\circ}\right)^{\prime}=\left(e^{\circ}\right)^{* \circ}=e^{* \circ}=1_{S}^{\circ}=1_{S}$, by Proposition 4.2 (ii).

Remark If $\rho$ (resp. $\sigma$ ) denotes the corresponding group congruence on $S$ (resp. $S^{\circ}$ ) then $\sigma=\left.\rho\right|_{S^{0}}$, i.e., $\sigma$ is the restriction of $\rho$ to $S^{\circ} \subseteq S$. In fact, every $\rho$-class $a \rho \in S / \rho$ contains the regular element $\bar{a}=a a^{*} a$ (see Lemma 4.1): since $\bar{a} \leq_{S} a$ and since by [3], Lemma 2.1, $a \rho \subseteq S$ is a principal order ideal of $\left(S, \leq_{S}\right), \bar{a} \in a \rho$. Furthermore, $\left.\rho\right|_{S^{\circ}}$ is a group congruence on $S^{\circ}$, because for 
any $a \rho=\bar{a} \rho \in G=S / \rho$ there exists $b \rho=\bar{b} \rho \in G$ such that $(a \rho)(b \rho)=1_{G}$, i.e., $(\bar{a} \rho)(\bar{b} \rho)=1_{G}$. Since by Lemma $3.1,1_{S} \rho=1_{G}=\left(1_{S}\right]=1_{S} \sigma$ it follows by [1], Theorem 10.24, that $\left.\rho\right|_{S^{\circ}}=R_{1_{G}}=\sigma$, where $R_{1_{G}}$ denotes the Dubreil equivalence defined by the anticone $1_{G}=\left(1_{S}\right]$ - see [2] .

By Theorem 3.4, in every $F$-monoid $S$ the identity $1_{S} \in S$ is rightresiduated; in particular, $1_{S} \cdot t$ exists in $\left(S, \leq_{S}\right)$ for any $t \in R(S)$. Therefore, we obtain from Lemma 5.1 and Proposition 5.2:

Corollary 5.3 Let $S$ be an F-monoid. Then (i) $T=R(S)$ is an F-regular monoid; (ii) $S=A(T)$, the set of associates of elements in $T$; (iii) for any $t \in T, 1_{S} \cdot t=\max \left\{x \in S \mid t x \leq_{S} 1_{S}\right\}$ exists in $\left(S, \leq_{S}\right)$.

We will show the converse of Corollary 5.3. Recall that for any $a \in S$, $\left\langle 1_{S}: a\right\rangle=\left\{x \in S \mid a x \leq_{S} 1_{S}\right\}$ (see [3]).

Proposition 5.4 Let $S$ be a monoid such that (i), (ii), (iii) in Corollary 5.3 are satisfied. Then $S$ is an F-monoid.

Proof. We will prove that for any $a \in S, 1_{S}: a=\max \left\langle 1_{S}: a\right\rangle$ exists. Then the statement follows by Theorem 3.4.

Let $a \in S$; then by (ii), tat $=t$ for some $t \in T$. Therefore, at $\in E_{S} \subseteq R(S)$ and ata $\in R(S)=T$. Hence by (iii), $1_{S}$ : at and $1_{S}$. ata exist in $\left(S, \leq_{S}\right)$. We show that $1_{S}$ : ata $=1_{S} \cdot a$.

By definition, ata $\left(1_{S} \cdot a t a\right) \leq_{S} 1_{S}$, i.e., at $\cdot a\left(1_{S} \cdot a t a\right) \leq_{S} 1_{S}$ and therefore $a\left(1_{S}\right.$ :ata $\leq_{S} 1_{S}$ :at $=1_{S}$, by the proof of sufficiency of Lemma 4.4 in [3]. Thus, $1_{S} \cdot$ ata $\in\left\langle 1_{S} \cdot a\right\rangle$.

Let $x \in S$ be such that $x \in\left\langle 1_{S}\right.$ : $\left.a\right\rangle$; then $a x \leq_{S} 1_{S}$ and $a x \in E_{S}$ (by [8], Lemma 2.1). By the above, also at $\in E_{S}$; therefore $a t \cdot a x \in E_{S}$ (by (i), $T=R(S)$ is a $F$-semigroup, hence by [2], Proposition 3.7, $E_{S}=E_{T}$ is a subsemigroup of $T \subseteq S)$. Hence, ata $\cdot x \leq_{S} 1_{S}$ and $x \leq_{S} 1_{S}$. ata. It follows that $1_{S} \cdot$ at $a=\max \left\langle 1_{S} \cdot a\right\rangle$ in $\left(S, \leq_{S}\right)$, that is, $1_{S} \cdot a$ exists in $\left(S, \leq_{S}\right)$.

Combining Corollary 5.3 and Proposition 5.4 we obtain the following characterization of $F$-monoids:

Theorem 5.5 Let $S$ be a monoid. Then $S$ is an F-monoid if and only if

(i) $T=R(S)$ is an F-regular monoid,

(ii) $S=A(T)=\{x \in S \mid t x t=t$ for some $t \in T\}$,

(iii) for every $t \in R(S), 1_{S} \cdot t$ (resp. $\left.1_{S} \cdot t\right)$ exists in $\left(S, \leq_{S}\right)$.

Example Let $S$ be the $F$-monoid given in Example (3) of Section 2 with $S_{\mu}=(\mathbb{Z},+)$ and $S_{\omega}=\left(\mathbb{N}_{0},+\right)$. Then the pivot of $S$ is 0 and 
(i) $T=R(S)=S_{\mu} \cup\{0\}$ is an $F$-semigroup since the group $S_{\mu}$ is an $F$ semigroup with idempotent pivot $\xi=\overline{0}$; hence adjoining a new identity 0 , $R(S)$ is an $F$-semigroup (by Proposition 2.1);

(ii) $S=A(T)$ : for every $n \in S_{\omega}=\mathbb{N}_{0}, t=-\bar{n} \in T=\mathbb{Z}$ satisfies $t+n+t=$ $(-\bar{n})+n+(-\bar{n})=-\bar{n}+\bar{n}-\bar{n}=-\bar{n}=t$; for every $a \in S_{\mu}=\mathbb{Z}, t=-a \in T$ satisfies $t+a+t=t$;

(iii) for any $t \in R(S)=S_{\mu} \cup\{0\}, 0 \cdot t=n$ if $t=-\bar{n}$, and $=-\bar{n}$ if $t=\bar{n}$.

\section{$6 \quad F-*-m o n o i d s$}

Following [4] we call an $F$-monoid $S F$-*-monoid if $S$ satisfies the identity

$$
(a b)^{*}=b^{*} a^{*} \text { for all } a, b \in S,
$$

with respect to the $*$-operation $a^{*}=1_{S}: a(a \in S)$ considered in Section 4 . Concerning such monoids we first have for $S^{*}=\left\{a^{*} \in S \mid a \in S\right\}$ :

Lemma 6.1 Let $S$ be an F-monoid. Then the following hold:

(1) $S^{*}=\{x \in S \mid x$ is the greatest element of a $\rho$-class $\}$

$$
=\left\{m \in S \mid m \text { is maximal in }\left(S, \leq_{S}\right)\right\} ;
$$

(2) $(a b)^{*}=b^{*} a^{*}$ for all $a, b \in S$ if and only if $S^{*}$ is a subsemigroup of $S$;

(3) If $S$ is an $F$-*-monoid then $S^{*}=H_{1}$, the group of units of $S$. In particular, $\left(a^{*}\right)^{-1}=a^{* *}$ for any $a^{*} \in S^{*}$.

Proof. The three statements are proved as in [4], Lemma 6.3. The only two points to be observed are the following. In (3), $H_{1} \subseteq S^{*}$ since for any $x \in H_{1}, x^{-1} \in H_{1}$ is a regular element of $S$; hence by [4], Theorem 2.2, $x^{-1}=$ $x^{-1}\left(1_{S}: x^{-1}\right) x^{-1}=x^{-1}\left(x^{-1}\right)^{*} x^{-1}$, i.e., $x=\left(x^{-1}\right)^{*} \in S^{*}$. Also in (3), if $a^{*} \in S^{*}=H_{1}$ then $a^{*} a \in E_{S}$ implies $\left(a^{*} a\right)^{*}=1_{S}$ (by Proposition 4.2 (ii)); therefore since $S$ is an $F$-*-monoid, $a^{*} a^{* *}=1_{S}$ and $a^{* *}=\left(a^{*}\right)^{-1}$.

Theorem 6.2 Let $S$ be an F-*-monoid. Then $S$ is regular.

Proof. Let $a \in S$; then by Lemma $6.1(3),\left(a^{*}\right)^{-1}=a^{* *}$. Hence it follows from Proposition 4.3 (ix), that

$$
\bar{a}=a a^{*} a^{* *}=a a^{*}\left(a^{*}\right)^{-1}=a .
$$

Since by Lemma $4.1, \bar{a} \in S$ is regular so is $a \in S$.

By the characterization of $F$-regular ( $F$-inverse) $*$-semigroups given in [4], Theorems 6.4 and 6.5, we obtain from Theorem 6.2 
Corollary 6.3 (1) Let $S$ be a monoid. Then $S$ is an F-*-semigroup if and only if $S$ is a semidirect product of a band with identity by a group.

(2) Let $S$ be a monoid with commuting idempotents. Then $S$ is an F-*-semigroup if and only if $S$ is a semidirect product of a semilattice with identity by a group.

\section{References}

[1] A. Clifford, G. Preston: The algebraic theory of semigroups. Vol. II, Amer. Math. Soc. Surveys 7 (Providence, 1967).

[2] E. Giraldes, P. Marques-Smith, H. Mitsch: Generalized F-semigroups. Math. Bohemica 130 (2005), 203-220.

[3] E. Giraldes, P. Marques-Smith, H. Mitsch: F-semigroups - submitted.

[4] E. Giraldes, P. Marques-Smith, H. Mitsch: F-regular semigroups. J. Algebra 274 (2004), 491-510.

[5] T. E. Hall: Some properties of local subsemigroups inherited by larger semigroups. Semigroup Forum 25 (1982), 33-48.

[6] A. Fidalgo Maia, H. Mitsch: Constructions of trivially ordered semigroups. Pure Math. Appl.13 (2002), 359-371.

[7] H. Mitsch: A natural partial order for semigroups. Proc. Amer. Math. Soc. 97 (1986), 384-388.

[8] H. Mitsch: Semigroups and their natural partial order. Math. Slovaca 44 (1994), 445-462.

[9] H. Mitsch, M. Petrich: Basic properties of E-inversive semigroups. Comm. Algebra 28 (2000), 5169-5182.

[10] H. Mitsch, M. Petrich: Restricting idempotents in E-inversive semigroups. Acta Sci. Math. 67 (2001), 555-570.

[11] M. Petrich: Introduction to Semigroups. Merill, Columbus/Ohio (1973). 